\title{
Transient abnormal $Q$ waves during exercise electrocardiography
}

\author{
F F Alameddine, A M Zafari
}

Heart 2003;90:el (http://www.heartjnl.com/cgi/content/full/90/1/el)

Myocardial ischaemia during exercise electrocardiography is usually manifested by ST segment depression or elevation. Transient abnormal $Q$ waves are rare, as $Q$ waves indicate an old myocardial infarction. The case of a patient with exercise induced transient abnormal $Q$ waves is reported. The potential mechanisms involved in the development of such an abnormality and its clinical implications are discussed.

$\mathrm{P}$ hysicians reading stress ECGs are on the lookout for interesting findings. Q waves usually represent scar tissue, yet there are numerous causes for the development of abnormal $\mathrm{Q}$ waves that are not due to myocardial infarction. ${ }^{1}$ Limited information is available to explain readily the transient nature of Q waves induced by exercise. We present an ECG illustrating a less appreciated cause of Q wave.

\section{PATIENT PRESENTATION}

A 65 year old man was referred for an exercise treadmill test before starting elective exercise. His medical history and physical examination were unremarkable, with a resting heart rate of 67 beats/min and blood pressure of 130/ $90 \mathrm{~mm} \mathrm{Hg}$. A resting 12 lead ECG showed non-pathological Q waves (fig l, first row). The patient exercised for 8:37 minutes, reaching a workload of 10 metabolic equivalents according to a standard Bruce protocol. He achieved his age predicted maximum heart rate without symptoms (peak heart rate of 161 beats/min and blood pressure of 180/ $86 \mathrm{~mm} \mathrm{Hg}$ ). The test was terminated at maximum heart rate and no significant ECG changes were noted (fig 1, second row).

Two minutes into recovery, at a heart rate of 95 beats/min and blood pressure of $170 / 90 \mathrm{~mm} \mathrm{Hg}$, the ECG (fig l, third row) showed abnormal Q waves. There was a pronounced QS pattern in leads III and aVF replacing the pre-exercise $\mathrm{R}$ waves. These $\mathrm{Q}$ waves persisted until the end of recovery time (fig l, fourth row) and the patient remained asymptomatic. A post-exercise two dimensional transthoracic echocardiogram, recorded while the patient had persistent transient abnormal $\mathrm{Q}$ waves (TAQs), showed no regional wall motion abnormalities. The $\mathrm{Q}$ waves resolved and the $\mathrm{R}$ waves were restored two hours after exercise testing (fig l, fifth row). All of the tracings were obtained in the supine position except for the second panel at peak exercise when the patient was standing.

An exercise echocardiography was done one month later to assess reproducibility. No echocardiographic abnormalities were noted and ECG tracings again reproduced the TAQs, though with less amplitude (maximum of $7 \mathrm{~mm}$ in the first exercise treadmill test and $4 \mathrm{~mm}$ in the second). These abnormalities were noted at one minute into recovery and disappeared at the end of recovery. These TAQs were identical in each of the supine and standing ECGs at every time point.
A follow up exercise treadmill test with nuclear imaging was performed one month later. The patient achieved for the third time a workload of 10 metabolic equivalents while remaining asymptomatic. No evidence of myocardial ischaemia was noted confirming the previous findings.

\section{DISCUSSION}

The appearance of transient and the exaggeration of previously existing Q waves in leads V1-V3 has been reported in the literature. The mechanism remains elusive. The development of transient Q waves in leads II, III, and aVF during exercise has been reported in only one patient by Segers et $a l,{ }^{2}$ one patient by Greenspan et $a l^{3}{ }^{3}$ and three patients by Przybojewski et al. ${ }^{4}$ Coronary arteriograms were negative for obstructive coronary artery disease in those patients and the authors concluded that these Q waves were not diagnostic of underlying coronary artery disease..$^{2-4}$

The development of TAQs can be explained by three interdependent mechanisms. Firstly, we suggest that left ventricular axis alteration during hyperventilation and hyperinflation induced by exercise may cause a rightward shift in the frontal plane electrical QRS axis with subsequent development of Q waves in leads II, III, and aVF. Bruce et al ${ }^{5}$ suggested that exercise induced enhancement of ventricular diastolic filling would increase intramyocardial tension with potential axis alteration, resulting in abnormal Q waves.

Another mechanism may be a conduction delay caused by interruption of a branch of the conduction system, which may alter the initial electrical vectors leading, for example, to a transient heart rate dependent left anterior fascicular block and the development of Q waves in leads II, III, and aVF.

Finally, spasm induced hypoperfusion may result in transient metabolic standstill and ultrastructural changes to the point that balanced electrical activity is not generated in these portions of the left ventricle. ${ }^{6}$ The finding of abnormal contraction patterns of the left ventricle associated with Q waves with an improved response to glyceryl trinitrate suggests that ischaemia may induce electrical silence without infarction. ${ }^{7}$ In experimental myocardial ischaemia in dogs, Q waves appeared during coronary occlusion and disappeared within four minutes after restoration of blood flow. ${ }^{8}$

The transient nature and relative reproducibility of TAQs in the subsequent exercise stress studies in our patient, as well as the absence of echocardiographic and nuclear imaging evidence of inferior wall ischaemia, confirm that the exercise induced TAQs in our patient do not represent myocardial ischaemia or myocardial infarction. TAQs indicate a transient loss of electromotive force or inexcitability, which is not synonymous with tissue death. Therefore, there was no indication for further cardiac testing in this asymptomatic low risk patient.

We conclude that exercise induced Q waves that return to the resting value must initially be considered to be normal unless ischaemic heart disease is seriously considered. These Q waves reflect a temporary loss of action potential secondary 


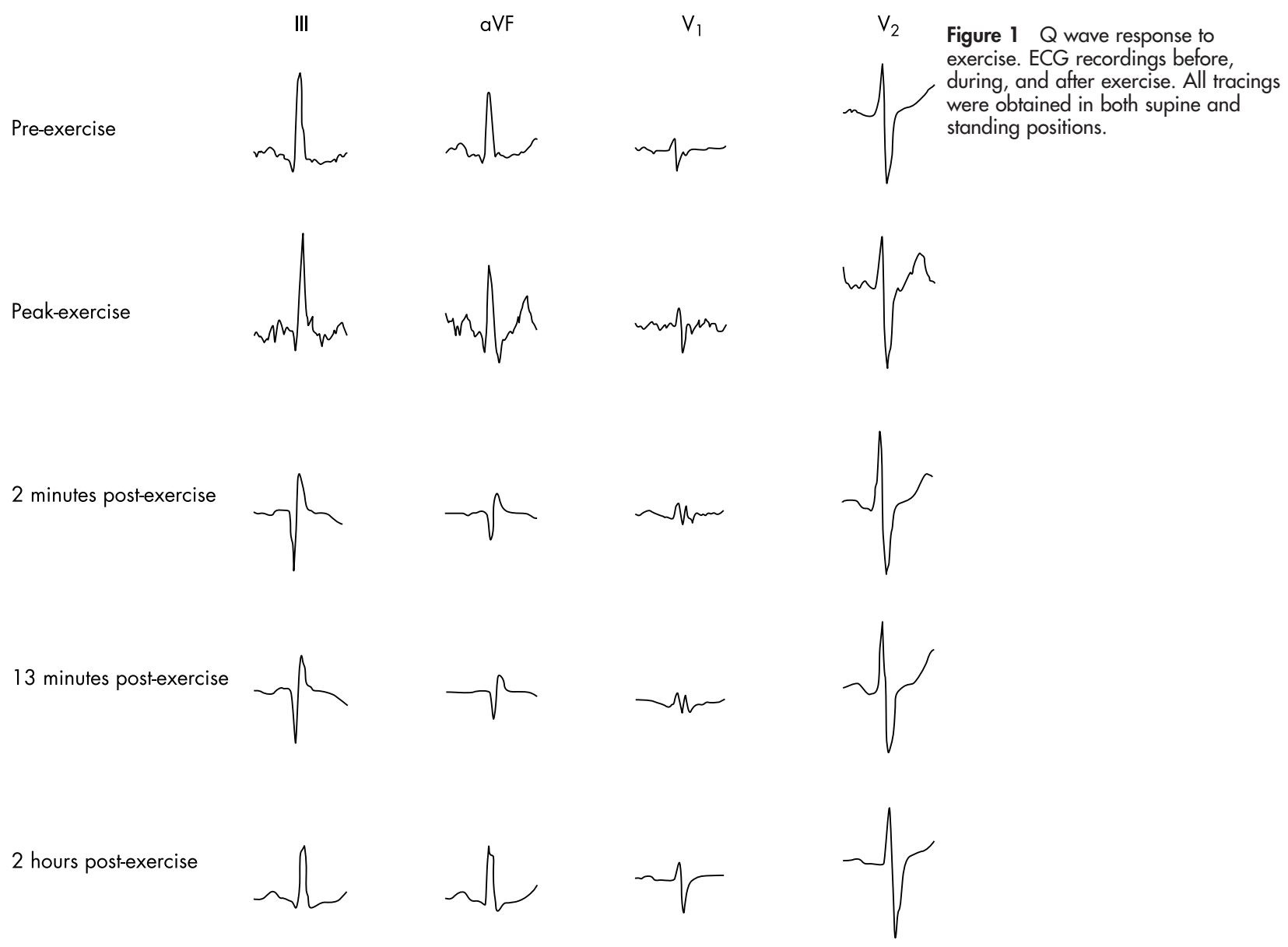

to electrical axis alteration, transient conduction block, or reversible ischaemia.

\section{ACKNOWLEDGEMENTS}

The authors thank Dr J Willis Hurst for his contribution in the preparation of this communication.

\section{Authors' affiliations}

F F Alameddine, A M Zafari*, Emory University School of Medicine, Division of Cardiology, Atlanta, Georgia, USA

*Also the Atlanta Veterans Affairs Medical Center, Decatur, Georgia, USA

Correspondence to: Dr A M Zafari, Emory University, Division of Cardiology, 1639 Pierce Drive, WMB 319, Atlanta, Georgia 30322, USA: azafari@emory.edu

Accepted 11 September 2003

\section{REFERENCES}

1 Hurst JW. Ventricular electrocardiography. Philadelphia: JB Lippincott, 1991:6.8-10.

2 Segers M, Regnier M, Delatte E. Alterations electrocardiographiques transitoires simulant les images coronariennes. Acta Cardiol 1951;6:39-52

3 Greenspan $M$, Anderson GJ. The significance of exercise-induced $Q$ waves. Am J Med 1979;67:454-9.

4 Przybojewski MB, Thorpe L. Transient pathological Q-waves occurring during exercise testing: assessment of their clinical significance in a presentation of a series of patients. J Electrocardiol 1987;20:121-30.

5 Bruce RA, Mazzarella JA, Jordan JW, et al. Quantitation of QRS and ST segment responses to exercise. Am Heart J 1966;71:455-66.

6 Bateman T, Gray R, Maddahi J, et al. Transient appearance of Q waves in coronary disease during exercise electrocardiography: consideration of mechanisms and clinical importance. Am Heart $J$ 1982; 104:182-4

7 Bodenheimer MM, Banka VS, Herman GA, et al. Reversible asynergy: histopathologic and electrocardiographic correlations in patients with coronary artery disease. Circulation 1976:53:792-6.

8 Gross H, Rubin IL, Laufer H, et al. Transient abnormal $Q$ waves in the dog without myocardial infarction. Am J Cardiol 1964;14:669-74. 